\title{
Editorial
}

\section{Approaching a New Year-And a New Look for the Journal}

In this last issue of the volume year, it is a good time to communicate some changes that we will be implementing to the journal. Some may be more welcome than others but all of them have been considered and discussed with the Editorial Board and ALA Production staff with the hope that it will provide a more streamlined process for the journal without compromising the experience of the readers or authors.

College $\mathcal{E}$ Research Libraries is a gold open access journal-as such, it is freely available. In fact, the journal does not retain copyright to the papers it publishes; it merely seeks permission from the authors to publish it. Thus, an author can indicate what kind of Creative Commons license they prefer.

Because it is freely available, there is no profit to be had and very few resources to expend on new bells and whistles. While there is not a climate of austerity, we are mindful of the cost-too often, I have seen databases or datasets that were once free move to a subscription or charge model for purposes of recovering costs and evolving the functions of the interface. So there are some decisions that we have made with the intent to have the journal run a little more smoothly and efficiently.

\section{No More Preprints}

Starting in January 2019, CERL will not be publishing preprints anymore. The original motivation for publishing the preprints is no longer a concern-at one time, it was 18 months from acceptance to publication and now it is less than a year. We are further committed to make it between 6 and 8 months from acceptance to publication.

The reasoning for this change is two-fold. It will help streamline the process in terms of the expenditure of effort and resources. The second issue is related to the versioning of the paper and the ephemeral status of the preprint. The preprint often gets harvested (and made discoverable) but it is not the copy of record. In addition, the preprint is not assigned a doi which has caused some access issues in the past.

As many authors are already uploading the accepted version of the article in their own institutional repositories, the articles are still discoverable.

\section{Taking Advantage of the Entire Page}

We are also going to be changing the layout of the articles in order to take advantage of more real estate on the page. When the journal moved online, it essentially approximated the print copy in an electronic format. The pdfs of the articles were almost identical to the print issue, including the scale and dimensions. The print version was very attractive and the online equivalent is as well - but there are features and changes that we can make to better communicate the author's work and enhance the reader's experience.

We will be moving to a letter size format which will allow charts and tables to be better oriented and presented. Right now, in order to fit on the page, charts and figures are shrunken, turned sidewise or may cut across pages. By almost doubling the page space, we hope to minimize those practices and display figures to their utmost advantage. Given that the journal is online only, most readers are reading online or printing out on letter size paper. 

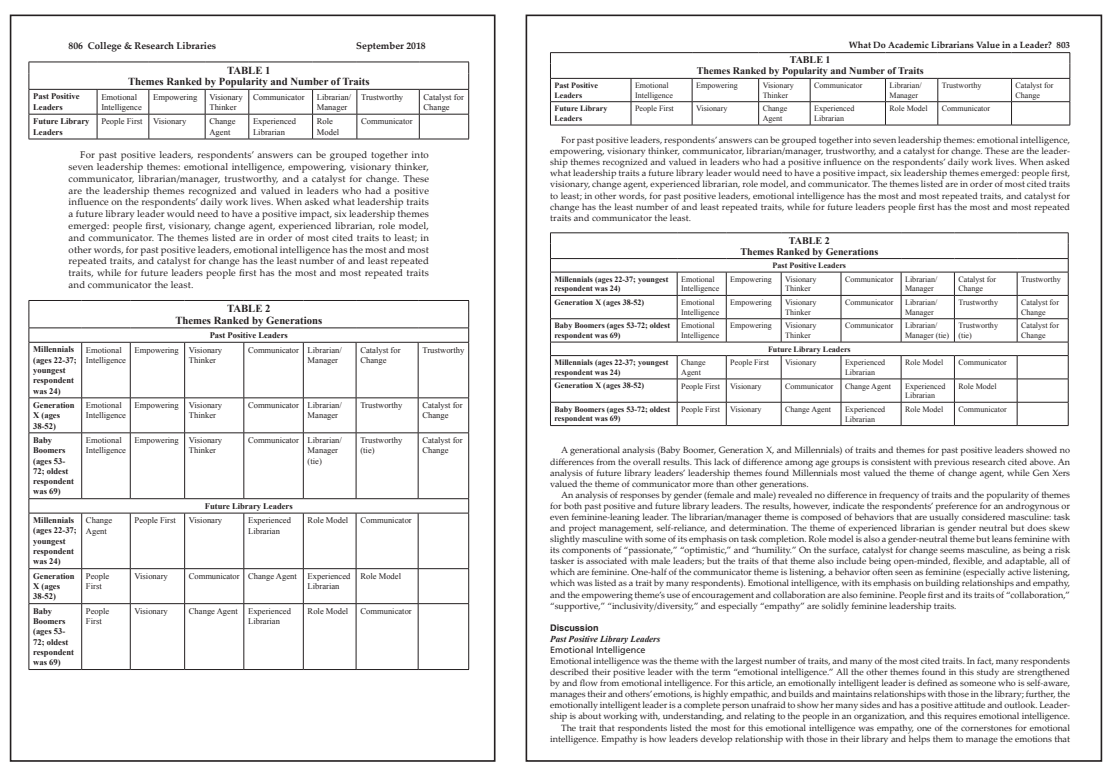

\section{$\leftarrow$ Before and After $\rightarrow$}

Images show how switching to letter-size layout will better utilize the space. Additional changes to the design are also anticipated.

\section{Template for Submissions}

We will also be adopting a submission template for authors to use when submitting to the journal. It is provided for authors to use for paper submissions, for a consistent font, margins, headings, etc. This template is also intended to offer information about format and guidelines, address questions about style and endnotes and provide guidance and reminders that may be useful to authors such as anonymizing the document before submission (with a separate document with a title page and author information) and embedded tables or figures in the document rather than putting them at the end or in another file altogether. The hope is that this effort will help authors in their submissions, provide a consistent format and presentation for reviewers as they read papers and, once an article is accepted, help minimize the time and effort involved in reformatting and copyediting.
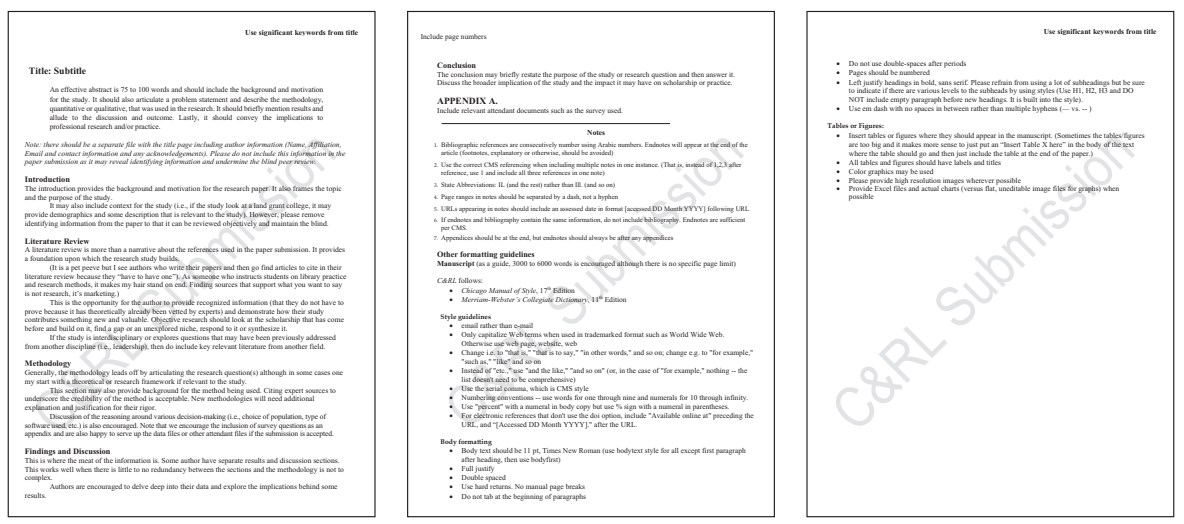

Template available at: https://tinyurl.com/yb99pfj3 


\section{Enhancing OJS}

The journal migrated it publication platform to Open Journal Systems last year; the submission and review platform was migrate this year. Going forward, our goal is to take advantage of other affordances of OJS and a digital environment. What exactly that will look like remains to be seen but we would like to be able to publish supplemental files for accepted such as datasets or other accompanying materials.

We don't make these changes lightly. They are intended to reduce wasted time and effort as well as provide a more transparent process and accommodating experience for stakeholders in CERL. However, I realize that it is a change and will take some getting used to - for all of us. 